PHYSICAL REVIEW B 96, 235407 (2017)

\title{
Linear ultrafast dynamics of plasmon and magnetic resonances in nanoparticles
}

\author{
Carlo Maria Lazzarini* and Levato Tadzio \\ Institute of Physics of the ASCR, ELI Beamlines Project, Na Slovance 2, 18221 Prague, Czech Republic \\ Jamie M. Fitzgerald \\ Department of Physics, Imperial College London, London SW7 2AZ, United Kingdom
}

José A. Sánchez-Gil

Instituto de Estructura de la Materia (IEM-CSIC), Consejo Superior de Investigaciones Cientificas, Serrano 121, 28006 Madrid, Spain

Vincenzo Giannini ${ }^{\dagger}$

Department of Physics, Imperial College London, London SW7 2AZ, United Kingdom

and Instituto de Estructura de la Materia (IEM-CSIC), Consejo Superior de Investigaciones Cientificas, Serrano 121, 28006 Madrid, Spain

(Received 8 August 2017; revised manuscript received 22 October 2017; published 5 December 2017)

\begin{abstract}
In this study we present an analytical description of the ultrafast localized surface plasmon and magnetic resonance dynamics in a single nanoparticle ( $\mathrm{Ag}$ or $\mathrm{Si}$ ), driven by an ultrashort (fs time scale) Gaussian pulse. Three possible scenarios have been found depending on the incident field, i.e., pulse duration much shorter than, similar to, and much longer than the localized surface plasmon resonance (LSPR) lifetime. A rich physics arises for $\tau_{\text {pulse }}<\tau_{\text {LSPR }}$, even in the linear regime. The surface plasmon dynamics is manifested as (i) a temporal delay of the surface plasmon excitation with regard to the freely propagating pulse and as (ii) a negative exponential tail after the exciting pulse is over. In addition, for sub-fs pulses clear oscillations in the near-field decay have been observed. A similar scenario has been observed considering a nonabsorbing Si sphere. Nanoparticle resonance dynamics may lead to a wealth of new phenomena and applications in nanophotonics such as multipole order resonance interference, pulse-induced delay or temporal shaping on the fs scale, high harmonic generation, attosecond near-field pulse sources, and electron acceleration from metasurface or 3D engineered nanostructures.
\end{abstract}

DOI: 10.1103/PhysRevB.96.235407

\section{INTRODUCTION}

The nanoplasmonics world is built around the fundamental concept of the collective resonant response of the conduction electrons of a nanostructure, known as plasmon resonances, induced by an incident electric field. Optical properties of plasmonic nanoparticles, such as their large optical cross sections and the enhancement of the optical near field in subwavelength regions, are well known in the literature [1,2].

The first theories [3-6] of light interacting with small objects (called later on plasmonics) based on classical electrodynamics go back more than a century. However, recent developments in nanofabrication techniques, high-sensitivity single-particle optical characterization techniques, and fast numerical modeling tools for simulating complex nanostructures have led to an increasing interest of the scientific community. This is mainly due to the huge number of applications nanoplasmonics can offer $[7,8]$. These range from solar cell efficiency improvement $[9,10]$ to surface-enhanced Raman spectroscopy [11], subdiffraction photon confinement [12,13], biosensing [14,15], nonlinear phenomena [16], nanoantennas for light-emitting devices [17-19], and quantum cascade lasers [20].

Silver and gold are the obvious choice among metals for many plasmon-based applications in the visible, because of the very narrow resonance. Moreover, plasmonic excitation

\footnotetext{
*carlomaria.lazzarini@eli-beams.eu

${ }^{\dagger}$ www.gianninilab.com
}

can be also obtained in the far-infrared zone using doped semiconductors [21,22] or in the ultraviolet zone using aluminium or silicon; in particular, the latter offers the possibility to excite ultraviolet long-range surface polaritons in extremely thin films [23]. However, metals present high nonradiative losses that lead to unwanted heating limiting some of their applications in photonics. Luckily, even though it is well known that interaction of the magnetic field part of light with matter is much weaker than the electric field one [24], lately, plasmoniclike resonances have been shown also in dielectrics. Indeed, nanoparticles made of high-refractive-index semiconductors (such as germanium, tellurium, GaAs, AlGaAs, GaP, and silicon) do not suffer from large intrinsic absorption at the visible, infrared, and telecom frequencies, thereby strongly attracting the attention and emerging as a promising alternative to plasmonic nanostructures for nanophotonic applications [25,26].

Inspired by the work of Garcia-Etxarri et al. [27] on dipolar magnetic resonances, as well as by the first theoretical and experimental demonstration of the magnetic resonance of $\mathrm{Si}$ nanoparticles in the visible and in the infrared range [28,28,29], substantial effort has been put into investigating the (multiorder) resonant properties of high-refractive-index nanoparticles [30-36], coining indeed the term of magnetic light [37]. The peculiar properties of such magnetic resonances, achieved with nonmagnetic, low-absorptive nanostructures, have been explored in a variety of fields: metamaterials [38-40], metasurfaces [41], nanoantennas [33,42,43], Fano resonances [44,45], and surface-enhanced spectroscopies [46,47]. Moreover, there is proof of the possibility to selectively tune the magnetic and electric dipole resonances in Si nanoparticles by changing 
their shape and aspect ratio and to calculate the scattering efficiencies of arbitrary shaped nanoparticles in the discrete dipole approximation [48]. The single Si nanoparticle dipole scattering, computed taking into account radiation correction terms in the magnetic dipole polarizability, has been calculated and found to be in agreement with the experimental scattering spectrum of laser-printed nanoparticles in a dark-field microscopic setup [49].

Recently, a different approach to nanophotonics has also emerged, considering all-dielectric metamaterials as completely transparent building blocks [50]. In these, light does not couple to plasmons or optical phonons, thus overcoming the critical issue of irreversible heat dissipation that was limiting many practical device applications.

Despite the intense research done in the field, most of these studies on plasmonics and magnetic light are limited to the monochromatic, continuous-wave regime. Nonetheless, in the last decades, due to the development of ultrashort (fs time scale) laser pulses, many attempts have been made to combine ultrashort laser pulses with nanostructures, generating the fast expanding field of ultrafast nanoplasmonics. In the latter, the key point is the control of the strong-field enhancement localized in space on the nanometer scale and in time on the fs and sub-fs scale, either in the linear or nonlinear regime. Different studies have been carried out, for example, on ultrafast fs [51] and as microscopy [52,53] or photoscopy [54], on sub-fs plasmon dynamics in tapered waveguides [55], on nanowires [56-59], and on silver nanoparticles [60,61]. Other new areas of research with increasing interest due to strong-field phenomena are high-order harmonic generation (HHG) [62-68], hard x-ray production from gold nanoplasma enhanced by prechirped ultrafast laser pulses [69], real-space coherent manipulation of electron motion in a single tunnel junction [70], and surface-plasmon-based particle acceleration [71-77].

Moreover, the surface plasmon propagation, the near field, and the far field scattered from planar surface defects have been extensively studied by Sánchez-Gil and colleagues [78,79].

However, an analytical description of the fields is still missing for nanoparticles. Experiments involving ultrafast pump-probe techniques and microscopy [80-82] are ahead of the theory, which mainly relies on the inadequate use of the physics of bulk material in order to explain nanoparticles or thin-film properties and response. For example, Makarov and colleagues [81] recently showed the possibility to tune the magnetic optical response of a $210 \mathrm{~nm}$ dielectric nanoparticle by ultrafast photoexcitation of dense electron-hole plasma, by changing the laser fluence. On the other hand, even the theory of the simplest case is unknown, i.e., the time dependence of near and far fields linearly induced in a small sphere by an ultrafast pulse.

Here we will address the latter configuration which, as we will see, is particularly interesting when plasmonic or magnetic resonances are driving the physics. In addition, there are many situations where nonlinear optical effects, coming from the tight focus of high laser intensities in nanoparticles, are just a correction of a few percent to the optical absorption [80,82]; this incidentally reveals the growing interest in this field and that the physics still relies on the linear response in most common situations.
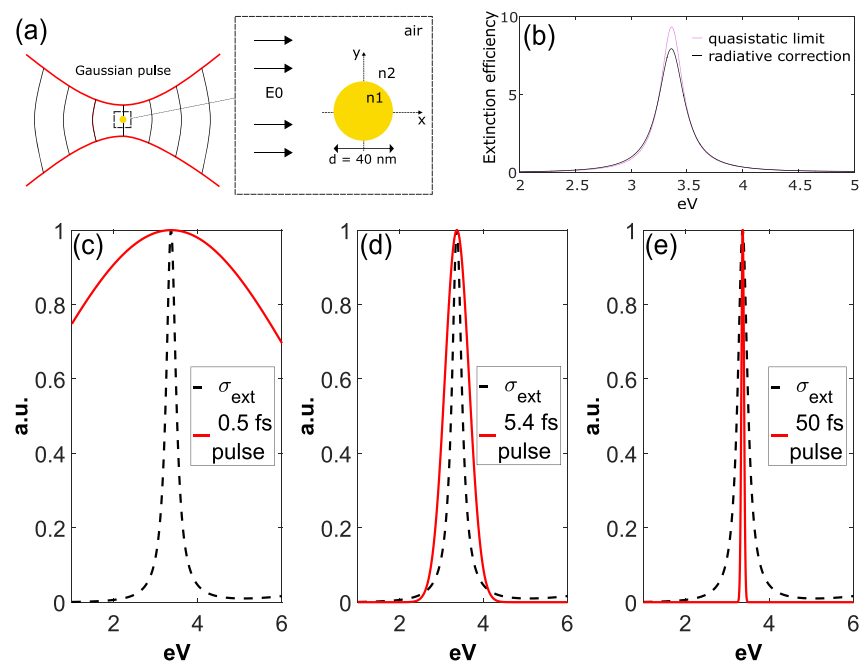

FIG. 1. (a) Scheme of the idea: a $40 \mathrm{~nm}$ diameter Ag nanoparticle placed in the beam waist of an incident Fourier-limited Gaussian pulse in the long-wavelength limit $(\lambda \gg d=2 a)$ under the paraxial approximation. In the beam waist the electric field is polarized along the $x$ axis. (b) Plane wave extinction efficiency of a $40 \mathrm{~nm}$ silver particle, computed considering radiative correction effect. (c), (d), (e) Normalized spectra of the laser pulse $\left(\left|E_{\text {inc }}\right|\right.$ in red) centered at $\lambda_{\mathrm{LSPR}}=369 \mathrm{~nm}$ having a bandwidth bigger, similar, or smaller compared to the LSPR one, considering a pulse duration respectively of $0.5 \mathrm{fs}, 5.4 \mathrm{fs}\left(\tau_{\mathrm{LSPR}}\right)$, and $50 \mathrm{fs}$.

We think that ultrafast dynamics is insightful, especially for the case of lower laser intensities or unfocused beams, below the fluence threshold for two-photon absorption. In this regard, we limit our analysis to the linear regime, presenting here an analytical description in time of the dynamics of a single nanoparticle response to an ultrafast driving pulse. Of course, it will be interesting to extend this study to the nonlinear case.

In the literature, spatially homogeneous monochromatic electric fields are usually considered. In this scenario the well known classical equations [83] for the optical properties of a single metal particle and the near-field enhancement are calculated considering plane waves.

In this article we show the response on a fs time scale of a single nanoparticle to a few-cycle Fourier-limited Gaussian ultrashort pulse in the long-wavelength limit. We consider the two cases of a $40 \mathrm{~nm}$ diameter silver sphere in the visible and a $460 \mathrm{~nm}$ diameter nonabsorbing silicon sphere in the near-IR.

In the next sections we will calculate from the single Ag particle the extinction curve [see Fig. 1(b)] the localized surface plasmon resonance (LSPR) lifetime [84], which turns out to be 5.4 fs. We will compute both analytically and numerically the near-field enhancement considering a driving pulse centered at a wavelength in the range 200 to $800 \mathrm{~nm}$, with a bandwidth much smaller than, comparable to, or broader than the LSPR curve (of time duration $\tau_{\text {pulse }}=$ $50 \mathrm{fs}, 5.4 \mathrm{fs}$, and $0.5 \mathrm{fs}$, respectively). We will then observe the ultrafast relaxation dynamics of the resonance-induced near-field enhancements and verify that a similar scenario happens in the bigger Si particle, solving on the fs time scale the electric and magnetic multipole particle response by Mie theory. 


\section{SILVER NANOPARTICLE IN DIPOLE LIMIT}

Here we present the results concerning the situation described in Fig. 1(a). A Fourier-limited fs Gaussian pulse impinges upon a single $40 \mathrm{~nm}$ diameter silver sphere. We solve this problem in the long-wavelength limit, i.e., when $k a \ll 1(k=2 \pi / \lambda$ is the wave vector, $\lambda$ is the wavelength, and $a$ is the particle radius). The silver is described by a Drude-Sommerfeld model; the dielectric function as a function of the probing frequency $\omega$ reads

$$
\varepsilon_{1}(\omega)=\varepsilon_{\infty}-\frac{\omega_{p}^{2}}{\omega^{2}+i \omega \gamma},
$$

where the dielectric function at infinite frequency, $\varepsilon_{\infty}=5$, accounts for the net contribution of positive ions. The plasma frequency $\hbar \omega_{p}=8.9 \mathrm{eV}$ is related to the effective mass of the electron $m^{*}$ and the electron density $N_{e}$ through $\omega_{p}=$ $\left(N_{e} e^{2} / \varepsilon_{0} m^{*}\right)^{1 / 2}$. The relaxation rate describes the effective electron scattering rate, with a corresponding relaxation time $\gamma^{-1}=17$ fs [85].

We have considered an incoming pulse polarized along the $x$ axis and propagating along $z$. Such pulse is a solution of the Helmholtz wave equation

$$
\nabla^{2} \mathbf{E}+k^{2} \mathbf{E}=0
$$

with $k=|\mathbf{k}|=\omega / c$. By using the paraxial approximation, the pulse can be written as the product of a spatial and a temporal part,

$$
\mathbf{E}(\mathbf{r}, \tau)=\mathbf{E}_{\text {space }}(\mathbf{r}) E_{\text {time }}(\tau) .
$$

Here $\mathbf{E}_{\text {space }}$ represents the solution of the paraxial Helmholtz equation, which is a Gaussian ( $T E M_{00}$ mode) wave propagating along $z$ and polarized along $x$. It is worth remarking that by considering the nanoparticle to be placed in the beam waist, the beam spot size is much bigger than the particle diameter; in other words we can consider the particle to be on the axis of the Gaussian pulse (i.e., having a radial coordinate $\rho \simeq 0$ ) and hence the pulse amplitude has constant value $E_{0}$ over the volume of the particle. In the adopted approximation, the spatial part of the solution can be considered to be a plane wave

$$
\mathbf{E}_{\text {space }}(\mathbf{r})=E_{0} e^{i k z} \hat{\mathbf{x}}
$$

where $E_{0}$ is a constant. The temporal part is given by a pulse with a Gaussian time profile of the form

$$
E_{\text {time }}(\tau)=e^{-\alpha_{p} \tau^{2}} e^{-i \omega_{0} \tau},
$$

where $\omega_{0}$ is the central pulse frequency and $\alpha_{p}$ is related to the pulse duration $\tau_{\text {pulse }}$, here in the range 0.5 to $250 \mathrm{fs}$, by $\alpha_{p}=4 \ln (2) / \tau_{\text {pulse }}^{2}$. The values of the pulse duration we consider cover the range of the state-of-the-art laser pulses for pump-probe experiments. Such values are useful to study the collective charge excitation and dephasing (1-10 fs), the nonthermal electron distribution effects, and the $e-e$ (or $e-h$ in a semiconductor) scattering (100 fs range) [82]. In addition, we found no physical behavior variation for longer or shorter pulses than the respectively longest or shortest cases here considered.
The next step is to expand the temporal part in Fourier components:

$$
E_{\text {time }}(\tau)=\frac{1}{2 \pi} \int_{-\infty}^{+\infty} \mathcal{E}(\omega) e^{-i \omega \tau} d \omega .
$$

For each frequency, the incident field on the small sphere becomes

$$
\mathbf{E}(\mathbf{r}, \omega)=E_{0} \mathcal{E}(\omega) \hat{\mathbf{x}} .
$$

The solution of an incident harmonic and uniform field on a small sphere is well known [19] and it is obtained from the electrostatic approximation. In fact, it reduces to the solution of the Laplace equation of the potential $\Phi$,

$$
\nabla^{2} \Phi=0
$$

where the electric field is given from the potential as $\mathbf{E}=$ $-\nabla \Phi$. The solution of the internal field has the following form (see Appendix):

$$
\begin{aligned}
\mathbf{E}_{1}(\omega) & =\mathbf{E}(\mathbf{r}, \omega) \frac{3 \varepsilon_{2}}{\varepsilon_{1}(\omega)+2 \varepsilon_{2}} \\
& =E_{0} \sqrt{\frac{\pi}{\alpha_{p}}} e^{-\frac{\left(\omega-\omega_{0}\right)^{2}}{4 \alpha_{p}}} \frac{3 \varepsilon_{2}}{\varepsilon_{\infty}+2 \varepsilon_{2}-\frac{\omega_{p}^{2}}{\omega(\omega+i \gamma)}} \hat{\mathbf{x}},
\end{aligned}
$$

where $\varepsilon_{2}$ is the background dielectric constant, which for simplicity will be considered to be air $\left(\varepsilon_{2}=1\right)$. The time response can be easily recovered thanks to the anti-Fourier transform of Eq. (9), as shown in the Appendix.

The induced dipolar moment by the field $\mathbf{E}(\omega)$ has value $\mathbf{p}=\varepsilon_{0} \varepsilon_{2} \alpha^{0}(\omega) \mathbf{E}(\omega)$, where $\alpha^{0}$ is the electrostatic polarizability. This last can be easily obtained [19] and represents a good approximation to $\alpha$ in the case of a small sphere:

$$
\alpha^{0}(\omega)=4 \pi \varepsilon_{0} a^{3} \frac{\varepsilon_{1}(\omega)-\varepsilon_{2}}{\varepsilon_{1}(\omega)+2 \varepsilon_{2}} .
$$

The electrostatic polarizability can be further improved considering the radiative correction $[83,86,87]$. In fact, imposing that the particle polarizability has to satisfy the optical theorem (see Appendix), we obtain a more accurate expression that reads

$$
\alpha(\omega)=\frac{\alpha^{0}(\omega)}{1-i \frac{k^{3}}{6 \pi \varepsilon_{0}} \alpha^{0}(\omega)} .
$$

From Eq. (11) it is straightforward to obtain the extinction cross section for a small particle as $[19,83]$

$$
\sigma_{\text {ext }}=\frac{k}{\varepsilon_{0}} \operatorname{Im}[\alpha(\omega)],
$$

with $\varepsilon_{0}$ the vacuum permittivity. For example, using the previous formulas, we calculated the extinction efficiency shown in Fig. 1(b). We can appreciate a FWHM of $0.245 \mathrm{eV}$, corresponding to a plasmon lifetime $\tau_{\mathrm{LSPR}}=5.4 \mathrm{fs}$.

Now we focus on the three following limit cases for the driving pulse: a pulse duration of $\tau_{\text {pulse }}=0.5,5.4$, and $50 \mathrm{fs}$ [Figs. 1(c)-1(e), respectively]. To solve the LSPR dynamics in the temporal domain, the electric field $E_{1}(t)$ has been calculated numerically, obtaining the $E$-field enhancement maps shown in Fig. 2 for the three limit cases of Fig. 1. The pulse central wavelength was spanned in the range 200 to 800 $\mathrm{nm}$.

We observed that, when the pulse bandwidth matches that of the LSPR [Fig. 2(b)], the 5.4 fs long pulse is delayed in 


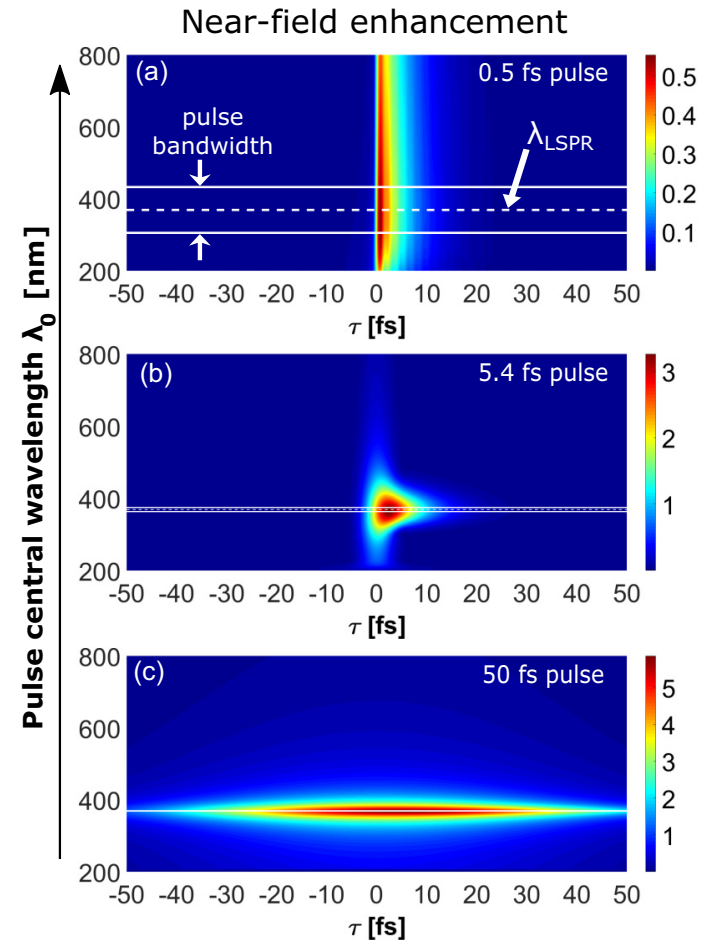

FIG. 2. Color maps showing the $E$-field enhancement $\left(\left|\mathbf{E}_{1}\right| / E_{0}\right)$ inside the $40 \mathrm{~nm} \mathrm{Ag}$ sphere on an ultrafast time scale. The plasmon resonance is excited with a Gaussian Fourier-limited pulse centered at $\lambda_{0}$ with a pulse duration of (a) $0.5 \mathrm{fs}$, (b) $5.4 \mathrm{fs}$, and (c) $50 \mathrm{fs}$. The dashed lines represent the resonance wavelength $\lambda_{\text {LSPR }}$, while the solid white lines show the pulse bandwidths.

the wavelength range around the resonance (dashed white line) with respect to the driving pulse. In the case in which the pulse bandwidth is narrower [Fig. 2(c)], it still excites the resonance (e.g., $E$-field enhancement $>1$ ) but the pulse shape and duration are not affected apart from a small time shift. Vice versa, in the case of a pulse much broader than the resonance [Fig. 2(a)], the resonant excitation effects in the pulse bandwidth region (identified by white lines) are not clearly observable.

The LSPR has been fully characterized by looking at the near-field decay in time after the driving pulse is over. Figure 3 shows in log scale the absolute values of the near-field normalized for the three cases, where their decays in time (black curve) are compared with the driving pulse Gaussian electric field (red dashed curve).

The first result, as a confirmation of the resonant excitation of plasmon modes in the particle, is the observation of a time-delayed exponential decay of the near field for driving pulses shorter than the LSPR lifetime $\tau_{\text {pulse }}=5.4$ fs [Figs. 3(a) and 3(b)]. The near-field enhancement decay has been fitted in the log scale obtaining a constant linear coefficient according to the Lorentz resonance mode decay constant $\Gamma / 2=0.186 \mathrm{fs}^{-1}$, which corresponds to the surface plasmon decay ( $\tau \simeq 5.37 \mathrm{fs}$ ). This is certainly true for pulses shorter than or comparable to the resonance, while for the $50 \mathrm{fs}$ case the fit presents a quadratic coefficient, indicating that there is no near-field LSPR-delayed decay, even if it still presents a Gaussian shape [Fig. 3(c)].
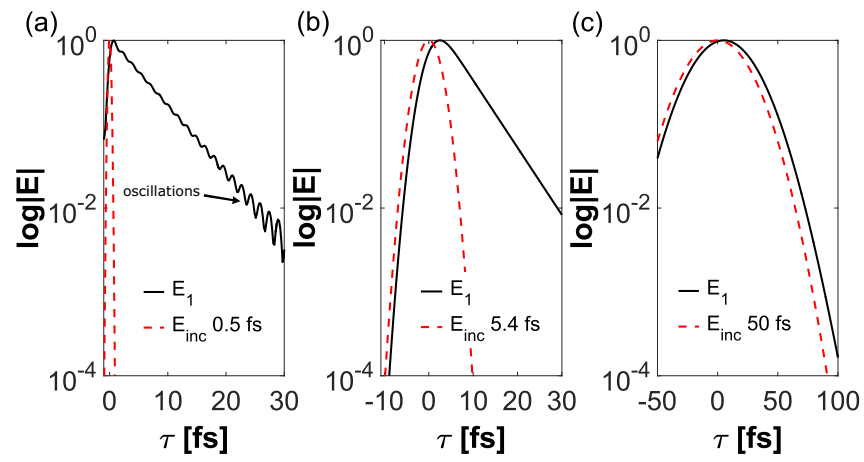

FIG. 3. $E$ field induced inside the $40 \mathrm{~nm}$ (diameter) $\mathrm{Ag}$ sphere (solid black line) by a laser pulse centered at the LSPR wavelength, $\lambda_{\text {LSPR }}=369 \mathrm{~nm}$, for different pulse durations: (a) $\tau_{\text {pulse }}=0.5 \mathrm{fs}$, (b) $5.4 \mathrm{fs}$, and (c) $50 \mathrm{fs}$. The absolute values of the electric fields have been normalized and plotted in a semilog scale in order to observe the shift of the LSPR-induced field with respect to the incoming one (dashed red line). For short pulses ( $\tau_{\text {pulse }} \leqslant \tau_{\text {LSPR }}=5.4 \mathrm{fs}$ ) it is clearly visible that the near field induced in the sphere is delayed, because of the resonant excitation, and decays as a Lorentz mode slower than the driving Gaussian pulse, showing ultrafast oscillations only for pulses much shorter than $\tau_{\text {LSPR }}$ [panel (a)].

The second important point, for all pulse durations tested, is the delay of the induced near field with respect to the driving pulse. This delay increases with pulse duration and saturates at a value of $\Delta t=5.4 \pm 0.1 \mathrm{fs}$ [Fig. 4(c)], which is in good agreement with the resonance lifetime $(\Gamma / 2)^{-1}=5.37 \mathrm{fs}$. On the other hand, for pulses shorter than $1 \mathrm{fs}$, the delay reaches a value of $\Delta t=0.7 \pm 0.1 \mathrm{fs}$ and the near-field enhancement is smaller than the unity. Indeed, in this scenario, the driving pulse has a bandwidth of a few hundred $\mathrm{nm}\left(\gg \Delta \lambda_{\mathrm{LSPR}}=\right.$ $27 \mathrm{~nm}$ ), meaning that the resonance has no time to build up.

Another confirmation that the above-mentioned delay indicates a resonant excitation of plasmon modes is the fact that, for a driving pulse centered out of resonance $(\lambda=800 \mathrm{~nm})$ with a bandwidth nonoverlapping with the plasmon resonance, the near field is perfectly matching the driving pulse-namely, a Gaussian shape with no delay, as can be seen from the blue curve in Fig. 4(b). The same situation happens for all the pulses whose duration is longer than $\tau_{\mathrm{LSPR}}=5.4 \mathrm{fs}$. On the other hand, for ultrashort sub-fs pulses, they are so broadband in the visible range that even if not matching the resonance they still induce a delayed decay as illustrated in Fig. 4(a) (where all the electric field amplitudes have been normalized for comparison).

We observed oscillations in the near-field decay by plotting its absolute value in time on a log scale. Their amplitude depends on the driving pulse duration, as they can be only seen in the log scale of Fig. 3(a), where the driving pulse is shorter than $1 \mathrm{fs}$.

The oscillation period does not depend on the driving pulse electric field or on the particle size, but only on the metal plasma parameters. Solving analytically the near field in time, the Lorentz-mode presents an exponential decay with constant 


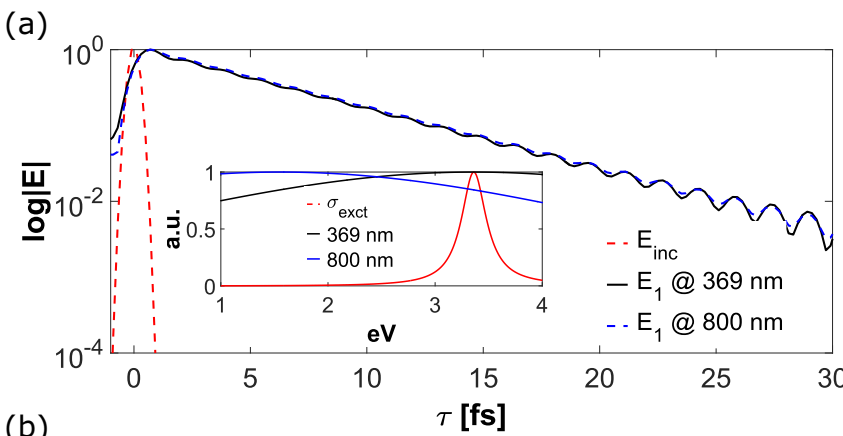

(b)

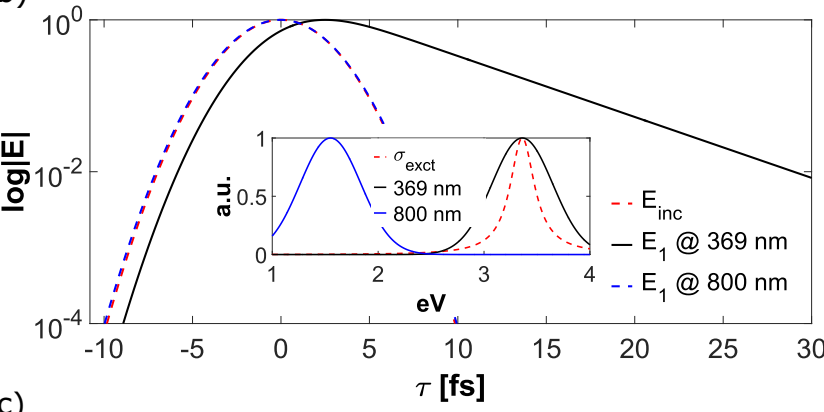

(c)

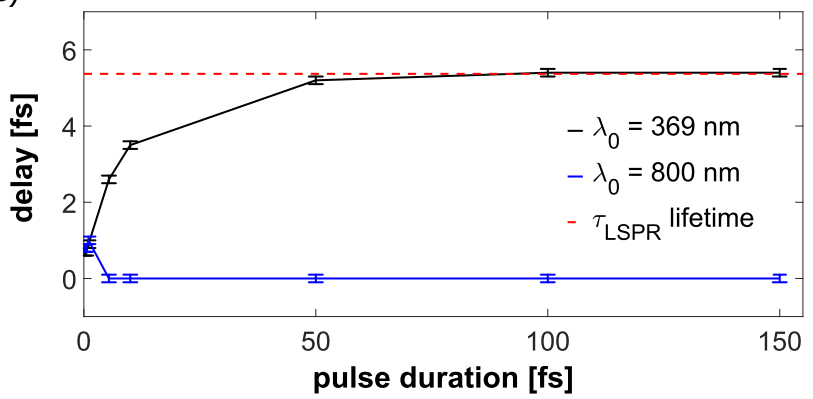

FIG. 4. Difference in exciting the LSPR with (a) a 0.5 fs ultrashort pulse and (b) a $5.4 \mathrm{fs}$ pulse. In both cases we show the $E_{1}(t)$ field inside the Ag particle excited with a pulse matching the resonance (solid black curve) and with a pulse of the same duration but out of resonance (dashed blue curve). The insets show the corresponding spectra of incoming pulses at and out of resonance compared to the resonance extinction curve (red). When pulses are really short (broadband), they are still able to excite the resonance as shown by the overlap of black and blue curves in (a). Vice versa when they have nonoverlapping bandwidth comparable to the resonance one; the $E$ field inside the sphere out of resonance, blue curve in (b), remains unchanged. (c) Delay of the $E$ field inside the sphere with respect to the incoming pulse as a function of pulse duration.

$\Gamma / 2$ and an oscillating part at a frequency

$$
\begin{aligned}
\tilde{\omega} & =\frac{\omega_{p}}{\sqrt{\varepsilon_{\infty}+2}} \sqrt{1-\frac{\gamma^{2}}{\omega_{p}^{2}} \frac{\varepsilon_{\infty}+2}{4}} \\
& =\omega_{\text {res }} \sqrt{1-\frac{\gamma^{2}}{4 \omega_{r e s}^{2}}}
\end{aligned}
$$

where $\omega_{\text {res }}=\frac{\omega_{p}}{\sqrt{\varepsilon_{\infty}+2}}$ is the resonance frequency satisfying the Fröhlich condition $\operatorname{Re}\left[\varepsilon_{1}\left(\omega_{R E S}\right)\right]=-2$. These ultrafast oscillations observed for the near-field decay of Fig. 3(a), for such a small nanoparticle, disappear if the radiation reaction effect is not considered in computing the effective polarizability. This means that the self-interacting correction is the origin of the oscillations in Figs. 3(a) and 4(a). This is confirmed by computing the electric dipole moment, by Mie theory, induced in the $\mathrm{Ag}$ sphere for a driving pulse of $0.5 \mathrm{fs}$ shown in Fig. 7(a).

\section{MIE THEORY FOR SILVER AND SILICON SUBWAVELENGTH SPHERES}

The single-particle dipole limit, $k a \ll 1$, where $k$ is the wave vector of the driving pulse and $a$ the particle radius, has been used so far to calculate nanoparticle near fields. However, in the case of bigger particles or different material we must consider the exact Mie solution $[87,88]$, in order to compute the particle polarizability and the cross sections, having so the possibility to consider retardation effects and multipole term contributions.

By using Mie coefficients (see Appendix), the particleinduced electric and magnetic dipole moments can be calculated as

$$
\begin{gathered}
\mathbf{p}(\omega)=\varepsilon_{0} \varepsilon_{2} \alpha_{E}(\omega) E_{0} \sqrt{\frac{\pi}{\alpha_{p}}} e^{-\frac{\left(\omega-\omega_{0}\right)^{2}}{4 \alpha_{p}}} \hat{x}, \\
\mathbf{m}(\omega)=\alpha_{M}(\omega) \frac{E_{0} \sqrt{\frac{\pi}{\alpha_{p}}} e^{-\frac{\left(\omega-\omega_{0}\right)^{2}}{4 \alpha_{p}}}}{c \mu_{0} \mu_{2}} \hat{y},
\end{gathered}
$$

where $\varepsilon_{0}$ and $\mu_{0}$ are the vacuum permittivity and permeability, $\mu_{2}$ is the relative permeability of the particle, and $\alpha_{E}$ and $\alpha_{M}$, respectively, are the effective electric and magnetic polarizabilities obtained by using the Mie expansion (see Appendix). In the time domain all the dipolar resonances have been calculated by an inverse Fourier transform (IFT) algorithm in MATLAB.

The long-wavelength limit $(k a \ll 1)$ of Mie terms also gives electric and magnetic dipole polarizabilities as in Eq. (11). Equivalent formulas for the magnetic polarizability in the same approximation can be found in the work of Zywietz et al. [49]. In fact, considering the effect of an incident uniform and harmonic magnetic field in the quasistatic approximation, they obtained

$$
\alpha_{M}^{0}(\omega)=-\frac{3 V}{2}\left[1-\frac{3}{\left(k_{1} a\right)^{2}}+\frac{3}{k_{1} a} \cot \left(k_{1} a\right)\right],
$$

and considering radiation correction terms

$$
\alpha_{M}^{R}(\omega)=\frac{9 V\left\{3\left[1-k_{1} a \cot \left(k_{1} a\right)\right]-\left(k_{1} a\right)^{2}\right\}}{\left(k_{1} a\right)^{2}\left(2 i k^{3} a^{3}+3 k^{2} a^{2}+6\right)-6\left(i k^{3} a^{3}+k^{2} a^{2}\right)\left[1-k_{1} a \cot \left(k_{1} a\right)\right]},
$$



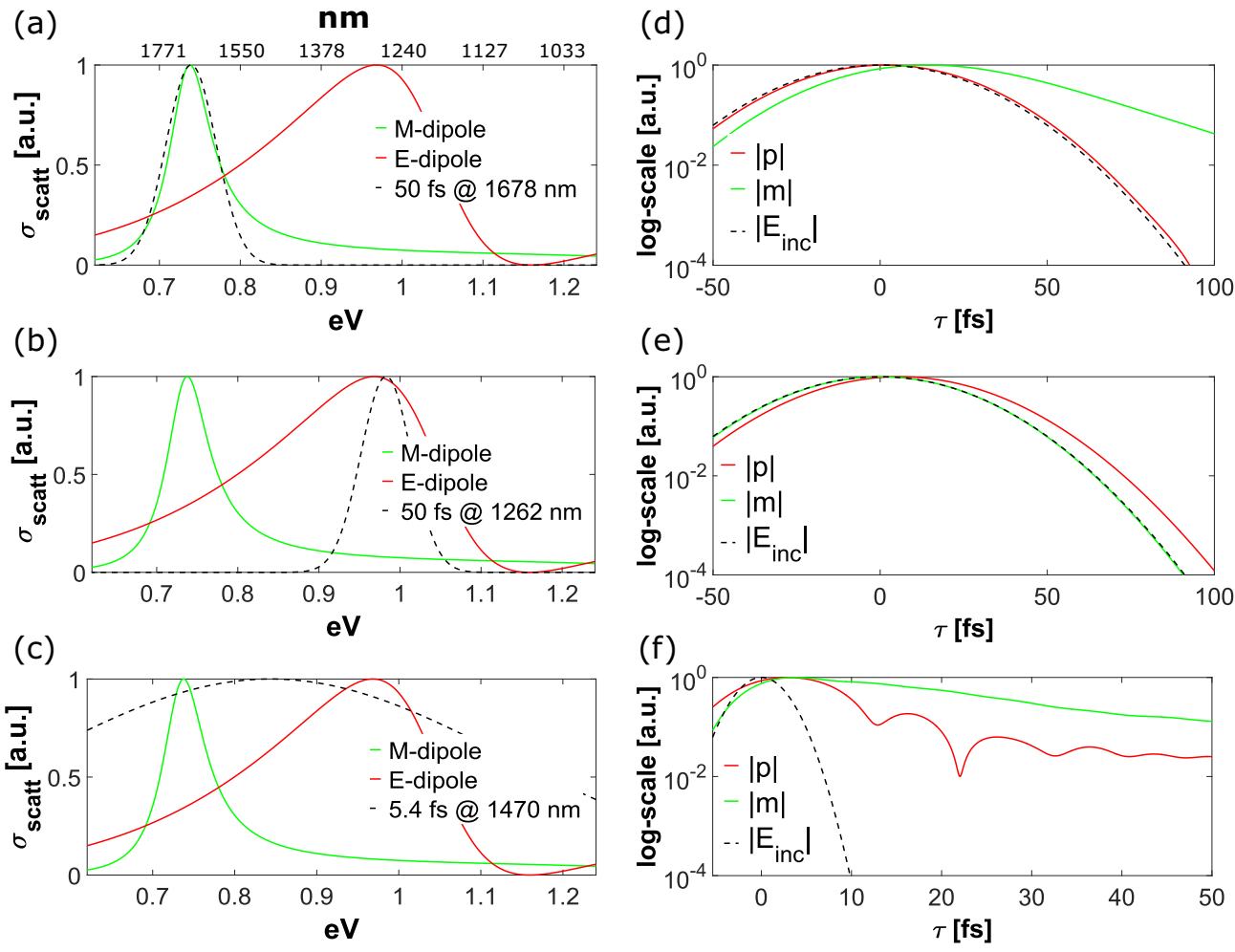

FIG. 5. (a), (b), (c) Spectral Gaussian bandwidths (dashed black curves), compared to electric (red) and magnetic (green) dipole resonance contributions to the scattering cross section for a $460 \mathrm{~nm} \mathrm{Si} \mathrm{sphere,} \mathrm{computed} \mathrm{by} \mathrm{Mie} \mathrm{theory,} \mathrm{of} \mathrm{the} \mathrm{following} \mathrm{pulses:} \mathrm{(a)} 50 \mathrm{fs}$ centered at $1678 \mathrm{~nm}$ (centered at the magnetic dipole resonance), (b) $50 \mathrm{fs}$ at $1262 \mathrm{~nm}$ (centered at the electric dipole resonance), and (c) $5.4 \mathrm{fs}$ pulse at $1470 \mathrm{~nm}$ (between the two dipoles). (d), (e), (f) Dipole moments $p(t)$ and $m(t)$ computed by the inverse Fourier transform, with the incident pulses respectively as in (a), (b), (c). Clearly visible are the delays of the dipole resonance contributions with respect to the driving pulse in case (d) and (e) where the resonances are selectively excited, while in case (f) the pulse is so broadband that even if centered between the two dipolar resonances, it still excites both with a similar delay.

with $V$ the volume of the particle, $k$ the wave number in free space $\left(k^{2}=\omega^{2} \mu_{0} \varepsilon_{0}\right)$, and $k_{1}$ the wave number inside the particle $\left(k_{1}^{2}=k^{2} \varepsilon_{1}\right)$.

By using Eq. (9) and the modified Drude-Sommerfeld model for the dielectric constant, we have found for the silver electric dipole moment $p(t)$ the same delayed decaying response to ultrafast pulses as for the near fields of Fig. 4 (Fig. 7). In the case of a $460 \mathrm{~nm}$ diameter nonabsorbing silicon sphere (with a refractive index of 3.5 as considered in [27]), the normalized dipole moments are shown in Fig. 5. For such a bigger particle with well-separated dipole resonances, a driving pulse with smaller bandwidth than these (50 fs long) can selectively excite the magnetic resonance [Fig. 5(a)] or the electric one [Fig. 5(b)], centered at a wavelength of 1678 or $1262 \mathrm{~nm}$, respectively. This is confirmed by looking at Figs. 5(d) and 5(e), where a clear delayed dipole response can be observed respectively for the magnetic (green curve) and for the electric resonance (red curve).

In the case of an ultrashort pulse centered between the two resonances [Fig. 5(c)], both dipole resonances are excited and the induced (normalized) dipoles present the same delay [Fig. 5(f)] even if with different decay coefficients. Incidentally, even though both (electric and magnetic) resonances exhibit a weakly asymmetric Fano line shape [89-91], pulse durations are such that their asymmetry is not fully probed; this will be shown elsewhere.
The delay in the Si particle response has been characterized as a function of both the driving pulse duration and the sphere diameter [Fig. 6(a)]. In the first case [Figs. 6(a), 6(b)] we
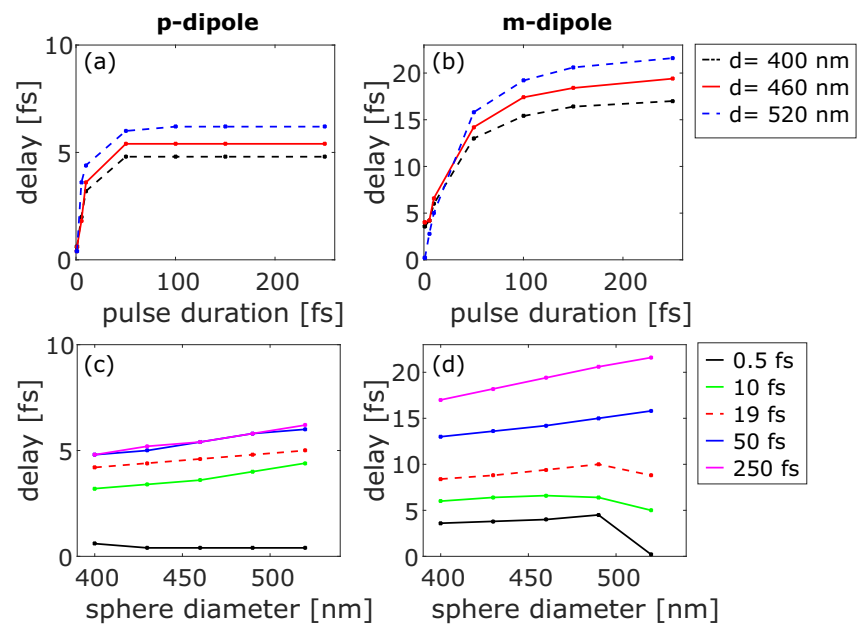

FIG. 6. Delay of the maximum of the electric (left columns) and magnetic (right column) dipole moments excited inside the sphere as a function of the incoming pulse duration for different sphere diameters [(a), (b)] and as a function of the sphere diameter for different pulse durations $[(\mathrm{c}),(\mathrm{d})]$. The Si refractive index is fixed at $n_{\mathrm{Si}}=3.5$. 
have a similar scenario to the Ag case, where the delays for the $p$ and $m$ dipole saturate at values in agreement with those expected from the resonance curve width $\Gamma$. Indeed, in the above-considered case of a $460 \mathrm{~nm}$ particle, we got $\Delta t_{p} \simeq(\Gamma / 2)^{-1}=5.2 \mathrm{fs}$ for the $p$ dipole and $\Delta t_{m}=19 \mathrm{fs}$ for the $m$ dipole. This is in agreement with the findings of Evlyukhin and colleagues [31], where they show that the induced magnetic-dipole mode lifetime of a Si nanoparticle decreases with increasing particle size (our case). Moreover, said mode becomes faster by increasing the dielectric constant of the surrounding medium; indeed if we consider the response of the same particle in water instead of air $\left(\varepsilon_{\text {water }}=1.73\right)$, we get a decreased resonance lifetime from 19 fs to $7 \mathrm{fs}$.

The dipolar mode response as a function of the nanoparticle diameter is shown in Figs. 6(c), 6(d). It is interesting to note that for a $50 \mathrm{fs}$ pulse, longer than the resonance lifetime, we have found a linear dependence for both the dipole response delays with the sphere diameter. Indeed the dipole response delay depends only on the particle dimension and dielectric refractive index, and can be simply explained by the traveling time inside the dielectric particle $\Delta t \simeq \frac{d n_{p}}{c}$. However, in the case in which the pulse is faster than the resonance and for bigger particles, we observed a smaller response delay. This can be explained taking into consideration the fact that for such short pulse duration, the equivalent spectrum is much broader than the two resonances and their relative separation, so the driving pulse is simultaneously exciting both resonances [as shown for example in Figs. 5(c), 5(f)]. In this scenario the measurement of a single resonance (dipole) mode with respect to the other starts to fail and the interplay of both modes (electric and magnetic) must be taken into account, as well as their interaction with themselves in such a broad spectrum (few eV).

\section{CONCLUSION}

We have investigated analytically and numerically the ultrafast localized surface plasmon and magnetic resonances dynamics in a single subwavelength nanoparticle (Ag or $\mathrm{Si}$ ), driven by a Gaussian ultrashort pulse ( 0.5 to 250 fs pulse duration).

We have characterized the effective extinction efficiency of a $40 \mathrm{~nm}$ diameter Ag sphere in the long-wavelength limit and solved the near-field enhancement inside the nanoparticle, by observing the resonant excitation of LSPR modes. These are manifested as (i) a temporal delay of the LSPR pulse with regard to the freely propagating pulse and (ii) as a negative exponential tail after the exciting pulse is over (for $\tau_{\text {pulse }}<$ $50 \mathrm{fs}$ ). Both the delay and the exponential $E$-field decay have resulted in agreement with the effective absorption efficiency from a modified Drude-Sommerfeld model for silver.

In the case of ultrashort driving pulses $\left(\tau_{\text {pulse }} \ll \tau_{\text {LSPR }}=\right.$ $5.4 \mathrm{fs}$ ) we have also observed ultrafast oscillations in the decaying part of the computed near field, much after the driving pulse is over. These are neither pulse nor particle size dependent, but they only depend on the plasma properties of the metal as shown by Eqs. (A21)-(A23), and they disappear if self-interacting radiation effects are not taken into account.

We have checked the consistency of our findings on the single Ag particle by considering a nonabsorbing silicon sphere of $460 \mathrm{~nm}$ diameter, where the exact Mie theory has been used to compute the first dipole moments. We observed a similar delayed response in the near field of the particle, where (either electric or magnetic) dipole resonances can be selectively excited with a 50 fs pulse.

We see as a possible development of this work the theoretical extension to the nonlinear case, when the high intensity (fluence) of the pumping laser could induce a significant change in the optical properties of metal and all-dielectric particles. Indeed, what has been shown in this work is valid in the linear case, easily implementable in an ultrafast laser laboratory (where usually the intensity is high and nonlinear effects are dominant) by attenuating the pulse energy or by changing the focusing condition in order to reduce the energy fluence on a single or different nanoparticles disposed on a substrate.

Under consideration are the study of the ultrafast (few fs) excitation and relaxation of collective motion of charge particles, which could be observable with state-of-the-art ultrafast pump-probe experiments, and the possibility to resolve the scattered far-field dynamics, and the laser-matter or laser-plasma interaction and evolution, on the same time scale. These possibilities could be achieved by exploiting some ultrafast phenomena (on the few fs or as time scale), for example by properly tuning the ultrafast oscillations we showed in the paper. Other interesting points not yet fully understood in the literature are the study of interference between different resonance orders in bigger particles and the multiple particle effects on the single-particle optical response whenever a pump beam is focused on a sample area containing several subwavelength nanostructures, for example nanoparticles fabricated on an array with spacing bigger than the nanoparticle dimension.

Moreover, the shown ability of nano-objects to introduce time delay in the propagation of ultrafast pulses along with the $E$-field enhancement can be used to generate, by means of properly engineered nanostructures, high harmonics and attosecond light sources with controlled (or delayed) temporal profiles. Finally, this research could be framed in the high-field plasmonics area where clustered gas targets and metamaterial surfaces are employed to exploit the direct acceleration of electrons or as innovative high-power laser-plasma diagnostics for particle acceleration experiments.

\section{ACKNOWLEDGMENTS}

Supported by the project ELI-Extreme Light Infrastructure, phase 2 (CZ.02.1.01/0.0/0.0/15_008/0000162), from the European Regional Development Fund and by the Ministry of Education, Youth, and Sports of the Czech Republic (Project No. LQ1606). J.A.S.-G. acknowledges the Spanish Ministerio de Economia y Competitividad for financial support through the grant LENSBEAM (FIS2015-69295-C3-2-P), and also Consejo Superior de Investigaciones Científicas (i-COOP LIGHT 2015CD0011).

\section{APPENDIX}

Considering incident radiation of wave vector $k=2 \pi / \lambda$ on a subwavelength nanoparticle in air whose polarizability 
is $\alpha$, the scattering and extinction efficiencies [see Fig. 1(b)] read [1]

$$
\begin{aligned}
\sigma_{\text {scatt }} & =\frac{k^{4}}{6 \pi \varepsilon_{0}^{2}}|\alpha(\omega)|^{2}, \\
\sigma_{\text {ext }} & =\frac{k}{\varepsilon_{0}} \operatorname{Im}[\alpha(\omega)],
\end{aligned}
$$

with $\varepsilon_{0}$ the vacuum permittivity. In the quasistatic limit the classical particle polarizability is

$$
\alpha^{0}(\omega)=4 \pi \varepsilon_{0} a^{3} \frac{\varepsilon_{1}(\omega)-\varepsilon_{2}}{\varepsilon_{1}(\omega)+2 \varepsilon_{2}},
$$

but this violates the optical theorem in the dipole limit [83] (i.e., no scattering is taken into account in computing the total extinction from a nanoparticle). Indeed, the optical theorem states that the total extinction cross section of a plane wave $\mathbf{E}_{p w}=E_{0} e^{i(k z-\omega t)} \hat{\mathbf{x}}$ propagating along the $z$ axis off an arbitrary object is

$$
\sigma_{\text {ext }}=\frac{4 \pi}{k^{2}} \operatorname{Re}[\mathbf{X} \cdot \hat{\mathbf{x}}],
$$

where $k$ is the wave vector, and the scattering amplitude $\mathbf{X}$ evaluated in the propagation direction (for $x, y=0, z \rightarrow \infty$ ) is related to the scattered far field

$$
\mathbf{E}_{S}=-\frac{e^{i k R}}{i k R} \mathbf{X} E_{0},
$$

with $R$ the radial distance from the scattering object. Now, considering that for a small particle the field $\mathbf{E}_{S}$ is originated from the dipole moment $\mathbf{p}$, and calculating the scattering and absorption cross sections, respectively, from the radiated power

$$
P_{\text {scatt }}=\frac{|\mathbf{p}|^{2} \omega^{4}}{12 \pi \varepsilon_{0} c^{3}}
$$

and the absorbed power

$$
P_{\mathrm{abs}}=\frac{\omega}{2} \operatorname{Im}\left[\mathbf{p} \cdot \mathbf{E} *_{0}\right],
$$

it turns out that the extinction cross section only accounts for the absorption and not for the scattering part. Therefore the quasistatic limit is not correct to compute the effective cross efficiencies of Eqs. (A1) and (A2), but radiation effects must be taken into account. Considering the radiation effect of an oscillating particle of charge $q$ interacting with itself it is possible to solve the equation of motion (r) where the radiation reaction force is given by the Abraham-Lorentz formula

$$
\mathbf{F}_{r}=\frac{q^{2} \dddot{\mathbf{r}}}{6 \pi \varepsilon_{0} c^{3}}
$$

with $c$ the speed of light in vacuum. This force induces a selffield $\mathbf{F}_{r}=q \mathbf{E}_{\text {self }}$, and thus we can calculate the induced dipole inside the sphere $\mathbf{p}=\alpha^{0}(\omega)\left(\mathbf{E}_{0}+\mathbf{E}_{\text {self }}\right)=\alpha(\omega) \mathbf{E}_{0}$, where the effective polarizability reads [83]

$$
\alpha(\omega)=\frac{\alpha^{0}(\omega)}{1-i \frac{k^{3}}{6 \pi \varepsilon_{0}} \alpha^{0}(\omega)} .
$$

The resonance curve for a $40 \mathrm{~nm} \mathrm{Ag}$ sphere [shown in Fig. 1(b)] has been obtained using Eq. (A2) and its induced electric dipole
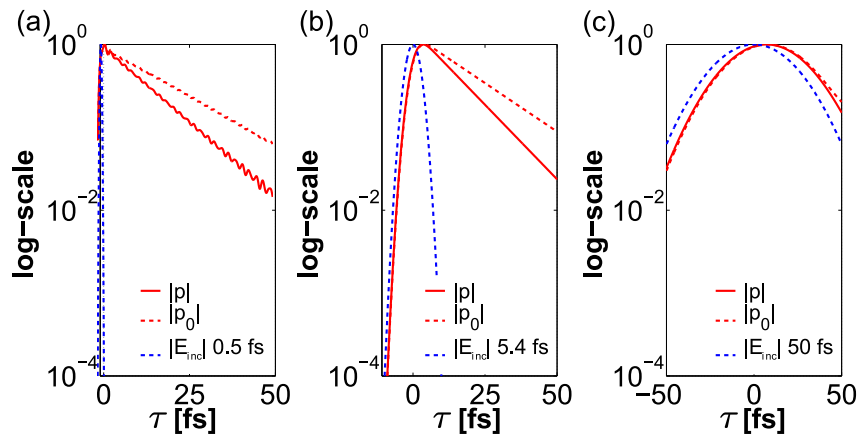

FIG. 7. Electric dipole moment induced in a single $40 \mathrm{~nm} \mathrm{Ag}$ particle, solved in time by inverse Fourier transform with (continuous red line) and without (dashed red) considering radiation effect, considering a driving pulse $\left(E_{\text {inc }}\right)$ of time duration (FWHM) of (a) $0.5 \mathrm{fs}$, (b) $5.4 \mathrm{fs}=\tau_{\mathrm{LSPR}}$, and (c) 50 fs. All moments have been calculated by using Mie coefficients and the modified DrudeSommerfeld model for the dielectric constant.

moment (shown in Fig. 7) by Fourier transforming the induced dipole in the frequency domain $\mathrm{p}(\omega)$. In order to retrieve the LSPR lifetime we can fit the extinction with a Lorentz-type curve

$$
L(\omega)=\frac{1}{\pi} \frac{\frac{\Gamma}{2}}{\left(\omega-\omega_{0}\right)^{2}+\left(\frac{\Gamma}{2}\right)^{2}},
$$

obtaining a resonance centered at $\hbar \omega_{0}=3.36 \mathrm{eV}$ and a FWHM $\Gamma=0.245 \mathrm{eV}$. The resonance lifetime, $\tau_{\mathrm{LSPR}}=\frac{2}{\Gamma}$, can be obtained by Fourier transform

$$
F^{-1} L(\omega)=\frac{1}{2 \pi} \int_{-\infty}^{\infty} L(\omega) e^{-i \omega t} d \omega=\frac{1}{2 \pi} e^{-i \omega_{0} t} e^{-\frac{|t|}{\tau_{\mathrm{LSPR}}}}
$$

If we consider a harmonic incident plane wave, the field induced inside the nanoparticle has the simple following dependence [83]:

$$
E_{1 p w}(\omega)=E_{0} \frac{3 \varepsilon_{2}}{\varepsilon_{1}(\omega)+2 \varepsilon_{2}}=E_{0} \frac{3 \varepsilon_{2}}{\varepsilon_{\infty}+2 \varepsilon_{2}-\frac{\omega_{p}^{2}}{\omega(\omega+i \gamma)}},
$$

where the subscript $p w$ stands for plane wave. This result can be generalized to a generic incident field making use of its Fourier expansion in the frequency domain

$$
E_{1}(\omega)=\mathcal{E}(\omega) \frac{3 \varepsilon_{2}}{\varepsilon_{1}(\omega)+2 \varepsilon_{2}},
$$

where

$$
\mathcal{E}(\omega)=\int_{-\infty}^{+\infty} E(t) e^{i \omega t} d t
$$

In the particular case of a Gaussian incident field [see Eq. (5)] the transformed near field reads

$$
E_{1}(\omega)=E_{0} \sqrt{\frac{\pi}{\alpha_{p}}} e^{-\frac{\left(\omega-\omega_{0}\right)^{2}}{4 \alpha_{p}}} \frac{3 \varepsilon_{2}}{\varepsilon_{\infty}+2 \varepsilon_{2}-\frac{\omega_{p}^{2}}{\omega(\omega+i \gamma)}} .
$$

Similarly, the $E$-field enhancement in time has been solved by the inverse Fourier transform of the near field computed in the 
frequency domain

$$
E_{1}(t)=F^{-1}\left[E_{1}(\omega)\right]=\frac{1}{2 \pi} \int_{-\infty}^{+\infty} E_{1}(\omega) e^{-i \omega t} d \omega .
$$

The color maps shown in Fig. 2 have been computed numerically in MATLAB by an inverse Fourier transform algorithm, with time steps of $0.0012 \mathrm{eV}$.

The time-dependent LSPR near field has been obtained analytically by looking at the inverse Fourier transform of Eq. (A16) as a convolution in time of two functions ( $f$ and $g$ ) that have been computed separately by inverse Fourier transform. That is,

$$
E_{1}(\tau)=F^{-1}[F(f) F(g)]=f * g=\int_{-\infty}^{\infty} f(t) g(\tau-t) d t,
$$

where the first term is the Gaussian incident field

$$
F(g)=E_{0} \sqrt{\frac{\pi}{\alpha_{p}}} e^{-\frac{\left(\omega-\omega_{0}\right)^{2}}{4 \alpha_{p}}},
$$

and the second is

$$
F(f)=\frac{3}{\varepsilon_{\infty}+2-\frac{\omega_{p}^{2}}{\omega(\omega+i \gamma)}} .
$$

So

$$
\begin{aligned}
f(t)= & F^{-1}[F(f)]=\frac{1}{2 \pi} \int_{-\infty}^{\infty} \frac{3 e^{-i \omega t}}{\varepsilon_{\infty}+2-\frac{\omega_{p}^{2}}{\omega(\omega+i \gamma)}} d \omega \\
= & \frac{1}{2 \pi} \int_{-\infty}^{\infty} \frac{3 e^{-i \omega t}}{\left(\varepsilon_{\infty}+2\right)} d \omega \\
& +\frac{1}{2 \pi} \int_{-\infty}^{\infty} \frac{3 \omega_{p}^{2} e^{-i \omega t}}{\left(\varepsilon_{\infty}+2\right)^{2} \omega(\omega+i \gamma)-\left(\varepsilon_{\infty}+2\right) \omega_{p}^{2}} \\
= & \frac{3 \delta(t)}{\varepsilon_{\infty}+2}-\frac{3 \omega_{p}^{2} e^{-\gamma t / 2} \sin (\tilde{\omega} t)}{\left(\varepsilon_{\infty}+2\right)^{2} \tilde{\omega}},
\end{aligned}
$$

where the second integral of the Fourier has been calculated in the complex plane $(\omega \rightarrow z \in \mathbb{C})$ by using the residue theorem, $\delta(t)$ is the Dirac function, and

$$
\begin{aligned}
\tilde{\omega} & =\frac{1}{2} \sqrt{\frac{4 \omega_{p}^{2}-\gamma^{2}\left(\varepsilon_{\infty}+2\right)}{\varepsilon_{\infty}+2}} \\
& \simeq \frac{\omega_{p}}{\sqrt{\varepsilon_{\infty}+2}}\left(1-\frac{\varepsilon_{\infty}+2}{\omega_{p}^{2}} \frac{\gamma^{2}}{8}\right)=\omega_{\mathrm{res}}-\frac{\gamma^{2}}{8 \omega_{\mathrm{res}}} .
\end{aligned}
$$

The inverse Fourier of Eq. (A18), $F^{-1}[g(\omega)](t)$, is simply the incident Gaussian field in time

$$
g(t)=E_{0} e^{-\alpha_{p} t^{2}} e^{-i \omega_{0} t} .
$$

The near field is then obtained by the convolution of Eq. (A17) as

$$
\begin{aligned}
E_{1}(\tau)= & \frac{3 E_{0}}{\varepsilon_{\infty}+2} e^{-\alpha_{p} \tau^{2}} e^{-i \omega_{0} \tau}-\frac{3 E_{0} \omega_{p}^{2}}{\left(\varepsilon_{\infty}+2\right)^{2} \tilde{\omega}} \\
& \times \int_{-\infty}^{\infty} e^{-\gamma t / 2} \sin (\tilde{\omega} t) e^{-\alpha_{p}(\tau-t)^{2}} e^{-i \omega_{0}(\tau-t)} d t \\
= & \frac{3 E_{0}}{\varepsilon_{\infty}+2} e^{-\alpha_{p} \tau^{2}} e^{-i \omega_{0} \tau} \\
& -\sqrt{\frac{\pi}{\alpha_{p}}} \frac{3 i E_{0} \omega_{p}^{2}}{2\left(\varepsilon_{\infty}+2\right)^{2} \tilde{\omega}} e^{-\gamma \tau / 2} e^{-i \tilde{\omega} \tau} \\
& \times\left[e^{\frac{-\gamma^{2}-4\left(\tilde{\omega}-\omega_{0}\right)^{2}+4 i \gamma\left(\tilde{\omega}-\omega_{0}\right)}{16 \alpha_{p}}}-e^{\frac{-\gamma^{2}-4\left(\tilde{\omega}+\omega_{0}\right)^{2}-4 i \gamma\left(\tilde{\omega}+\omega_{0}\right)}{16 \alpha_{p}}} e^{+i 2 \tilde{\omega} \tau}\right] .
\end{aligned}
$$

For the case of a nonabsorbing subwavelength Si sphere we have followed the same road, where the electric and magnetic polarizabilities in the quasistatic limit are

$$
\begin{aligned}
& \alpha_{E}^{0}(\omega)=-\frac{6 \pi}{k^{3}} \tan \left(\alpha_{1}\right), \\
& \alpha_{M}^{0}(\omega)=-\frac{6 \pi}{k^{3}} \tan \left(\beta_{1}\right),
\end{aligned}
$$

and the the effective polarizabilities are

$$
\begin{aligned}
& \alpha_{E}(\omega)=-\frac{\frac{6 \pi}{k^{3}} \tan \left(\alpha_{1}\right)}{1+i \tan \left(\alpha_{1}\right)}, \\
& \alpha_{M}(\omega)=-\frac{\frac{6 \pi}{k^{3}} \tan \left(\beta_{1}\right)}{1+i \tan \left(\beta_{1}\right)},
\end{aligned}
$$

with the extinction cross section

$$
\sigma_{\text {ext }}(\omega)=k \operatorname{Im}\left[\alpha_{E}+\alpha_{M}\right] .
$$

The Mie coefficients are $\alpha_{1}$ (proportional to the electric dipole) and $\beta_{1}$ (proportional to the magnetic dipole), where

$$
\begin{gathered}
\tan \left(\alpha_{n}\right)=\frac{m^{2} j_{n}(y)\left[x j_{n}(x)\right]^{\prime}-j_{n}(x)\left[y j_{n}(y)\right]^{\prime}}{m^{2} j_{n}(y)\left[x y_{n}(x)\right]^{\prime}-y_{n}(x)\left[y j_{n}(y)\right]^{\prime}}, \\
\tan \left(\beta_{n}\right)=\frac{j_{n}(y)\left[x j_{n}(x)\right]^{\prime}-j_{n}(x)\left[y j_{n}(y)\right]^{\prime}}{j_{n}(y)\left[x y_{n}(x)\right]^{\prime}-y_{n}(x)\left[y j_{n}(y)\right]^{\prime}},
\end{gathered}
$$

in terms of the spherical Bessel and Neumann functions of $n$ order $\left[j_{n}(x)\right.$ and $y_{n}(x)$, respectively], with relative refractive index $m=3.5$, size parameters $x=k a, y=m x$, and the prime indicating the derivative with respect to the argument $(x$ or $y)$.
[1] C. F. Bohren and D. R. Huffman, Absorption and Scattering of Light by Small Particles (John Wiley \& Sons, New York, 1993).

[2] W. L. Barnes, J. Opt. A: Pure Appl. Opt. 8, S87 (2006).
[3] Lord Rayleigh, Philos. Mag. 41, 107, 274, 447 (1871).

[4] Lord Rayleigh, Philos. Mag. 47, 375 (1899).

[5] G. Mie, Ann. Phys. 330, 377 (1908).

[6] R. H. Ritchie, Phys. Rev. 106, 874 (1957). 
[7] V. Giannini, A. I. Fernández-Domínguez, Y. Sonnefraud, T. Roschuk, R. Fernández-García, and S. A. Maier, Small 6, 2498 (2010).

[8] S. A. Maier, Plasmonics: Fundamentals and Applications (Springer, New York, 2007).

[9] P. Andrew, S. C. Kitson, and W. L. Barnes, J. Mod. Opt. 44, 395 (1997).

[10] M. Westphalen, U. Kreibig, J. Rostalski, H. Lüth, and D. Meissner, Sol. Energy Mater. Sol. Cells 61, 97 (2000).

[11] P. L. Stiles, J. A. Dieringer, N. C. Shah, and R. P. Van Duyne, Annu. Rev. Anal. Chem. 1, 601 (2008).

[12] J. D. Caldwell, O. J. Glembocki, Y. Francescato, N. Sharac, V. Giannini, F. J. Bezares, J. P. Long, J. C. Owrutsky, I. Vurgaftman, J. G. Tischler, V. D. Wheeler, N. D. Bassim, L. M. Shirey, R. Kasica, and S. A. Maier, Nano Lett. 13, 3690 (2013).

[13] J. M. Pitarke, V. M. Silkin, E. V. Chulkov, and P. M. Echenique, Rep. Prog. Phys. 70, 1 (2007).

[14] J. Homola, S. S. Yee, and G. Gauglitz, Sens. Actuators, B 54, 3 (1999).

[15] M. L. Coluccio, M. Francardi, F. Gentile, P. Candeloro, L. Ferrara, G. Perozziello, and E. Di Fabrizio, Opt. Laser Eng. 76, 45 (2016).

[16] M. Kauranen and A. V. Zayats, Nat. Photonics 6, 737 (2012).

[17] J. A. Schuller, E. S. Barnard, W. Cai, Y. C. Jun, J. S. White, and M. L. Brongersma, Nat. Mater. 9, 193 (2010).

[18] M. S. Tame, K. R. McEnery, S. K. Özdemir, J. Lee, S. A. Maier, and M. S. Kim, Nat. Phys. 9, 329 (2013).

[19] V. Giannini, A. I. Fernández-Domínguez, S. C. Heck, and S. A. Maier, Chem. Rev. 111, 3888 (2011).

[20] A. Tredicucci, C. Gmachl, F. Capasso, A. L. Hutchinson, D. L. Sivco, and A. Y. Cho, Appl. Phys. Lett. 76, 2164 (2000).

[21] V. Giannini, A. Berrier, S. A. Maier, J. A. Sánchez-Gil, and J. G. Rivas, Opt. Express 18, 2797 (2010).

[22] M. P. Fischer, C. Schmidt, E. Sakat, J. Stock, A. Samarelli, J. Frigerio, M. Ortolani, D. J. Paul, G. Isella, A. Leitenstorfer, P. Biagioni, and D. Brida, Phys. Rev. Lett. 117, 047401 (2016).

[23] V. Giannini, Y. Zhang, M. Forcales, and J. G. Rivas, Opt. Express 16, 19674 (2008).

[24] L. Landau, L. Pitaevskii, and E. Lifshitz, Electrodynamics of Continuous Media, 2nd ed. (Pergamon Press, Oxford, UK, 1984).

[25] M. Khorasaninejad, W. T. Chen, R. C. Devlin, J. Oh, A. Y. Zhu, and F. Capasso, Science 352, 1190 (2016).

[26] A. I. Kuznetsov, A. E. Miroshnichenko, M. L. Brongersma, Y. S. Kivshar, and B. Lukyanchuk, Science 354, aag2472 (2016).

[27] A. García-Etxarri, R. Gómez-Medina, L. S. Froufe-Pérez, C. López, L. Chantada, F. Scheffold, J. Aizpurua, M. NietoVesperinas, and J. J. Sáenz, Opt. Express 19, 4815 (2011).

[28] K. Vynck, D. Felbacq, E. Centeno, A. I. Căbuz, D. Cassagne, and B. Guizal, Phys. Rev. Lett. 102, 133901 (2009).

[29] A. B. Evlyukhin, C. Reinhardt, A. Seidel, B. S. Luk'Yanchuk, and B. N. Chichkov, Phys. Rev. B 82, 045404 (2010).

[30] J. M. Geffrin, B. García-Cámara, R. Gómez-Medina, P. Albella, L. S. Froufe-Pérez, C. Eyraud, A. Litman, R. Vaillon, F. González, M. Nieto-Vesperinas, J. J. Sáenz, and F. Moreno, Nat. Commun. 3, 1171 (2012).

[31] A. B. Evlyukhin, S. M. Novikov, U. Zywietz, R. L. Eriksen, C. Reinhardt, S. I. Bozhevolnyi, and B. N. Chichkov, Nano Lett. 12, 3749 (2012).
[32] S. Person, M. Jain, Z. Lapin, J. J. Sáenz, G. Wicks, and L. Novotny, Nano Lett. 13, 1806 (2013).

[33] I. Staude, A. E. Miroshnichenko, M. Decker, N. T. Fofang, S. Liu, E. Gonzales, J. Dominguez, T. S. Luk, D. N. Neshev, I. Brener, and Y. Kivshar, ACS Nano 7, 7824 (2013).

[34] T. G. Habteyes, I. Staude, K. E. Chong, J. Dominguez, M. Decker, A. Miroshnichenko, Y. Kivshar, and I. Brener, ACS Photonics 1, 794 (2014).

[35] A. B. Evlyukhin, R. L. Eriksen, W. Cheng, J. Beermann, C. Reinhardt, A. Petrov, S. Prorok, M. Eich, B. N. Chichkov, and S. I. Bozhevolnyi, Sci. Rep. 4, 4126 (2014).

[36] U. Zywietz, M. K. Schmidt, A. B. Evlyukhin, C. Reinhardt, J. Aizpurua, and B. N. Chichkov, ACS Photonics 2, 913 (2015).

[37] A. I. Kuznetsov, A. E. Miroshnichenko, Y. H. Fu, J. Zhang, and B. Luk'yanchuk, Sci. Rep. 2, 492 (2012).

[38] R. Paniagua-Domínguez, F. Lopez-Tejeira, R. Marqués, and J. A. Sánchez-Gil, New J. Phys. 13, 123017 (2011).

[39] J. C. Ginn, I. Brener, D. W. Peters, J. R. Wendt, J. O. Stevens, P. F. Hines, L. I. Basilio, L. K. Warne, J. F. Ihlefeld, P. G. Clem, and M. B. Sinclair, Phys. Rev. Lett. 108, 097402 (2012).

[40] R. Paniagua-Domínguez, D. R. Abujetas, and J. A. Sánchez-Gil, Sci. Rep. 3, 1507 (2013).

[41] R. Paniagua-Domínguez, Y. F. Yu, A. E. Miroschnichenko, L. A. Krivitsky, Y. H. Fu, V. Valuckas, L. Gonzaga, Y. T. Toh, A. Y. S. Kay, B. Luk'yanchuk, and A. I. Kuznetsov, Nat. Commun. 7, 10362 (2016).

[42] M. K. Schmidt, R. Esteban, J. J. Sáenz, I. Suárez-Lacalle, S. Mackowski, and J. Aizpurua, Opt. Express 20, 18609 (2012).

[43] A. E. Krasnok, A. E. Miroshnichenko, P. A. Belov, and Y. S. Kivshar, Opt. Express 20, 20599 (2012).

[44] B. Hopkins, D. S. Filonov, A. E. Miroshnichenko, F. Monticone, A. Alù, and Y. S. Kivshar, ACS Photonics 2, 724 (2015).

[45] D. R. Abujetas, M. A. G. Mandujano, E. R. Mendez, and J. A. Sanchez-Gil, ACS Photonics 4, 1814 (2017).

[46] P. Albella, R. A. De La Osa, F. Moreno, and S. A. Maier, ACS Photonics 1, 524 (2014).

[47] I. Staude, V. V. Khardikov, N. T. Fofang, S. Liu, M. Decker, D. N. Neshev, T. S. Luk, I. Brener, and Y. S. Kivshar, ACS Photonics 2, 172 (2015)

[48] A. B. Evlyukhin, C. Reinhardt, and B. N. Chichkov, Phys. Rev. B 84, 235429 (2011).

[49] U. Zywietz, A. B. Evlyukhin, C. Reinhardt, and B. N. Chichkov, Nat. Commun. 5, 3402 (2014).

[50] S. Jahani and Z. Jacob, Nat. Nanotechnol. 11, 23 (2016).

[51] A. Kubo, N. Pontius, and H. Petek, Nano Lett. 7, 470 (2007).

[52] M. I. Stockman, M. F. Kling, U. Kleineberg, and F. Krausz, Nat. Photonics 1, 539 (2007).

[53] F. Süßmann and M. F. Kling, Phys. Rev. B 84, 119911 (2011).

[54] M. Lupetti, J. Hengster, T. Uphues, and A. Scrinzi, Phys. Rev. Lett. 113, 113903 (2014).

[55] M. I. Stockman, Phys. Rev. Lett. 93, 137404 (2004).

[56] M. Sandtke and L. Kuipers, Nat. Photonics 1, 573 (2007).

[57] C. Rewitz, T. Keitzl, P. Tuchscherer, J. S. Huang, P. Geisler, G. Razinskas, B. Hecht, and T. Brixner, Nano Lett. 12, 45 (2012).

[58] M. Wulf, A. De Hoogh, N. Rotenberg, and L. Kuipers, ACS Photonics 1, 1173 (2014).

[59] T. P. H. Sidiropoulos, R. Röder, S. Geburt, O. Hess, S. A. Maier, C. Ronning, and R. F. Oulton, Nat. Phys. 10, 870 (2014).

[60] T. Brixner, F. J. García De Abajo, J. Schneider, and W. Pfeiffer, Phys. Rev. Lett. 95, 093901 (2005). 
[61] A. A. Unal, A. Stalmashonak, G. Seifert, and H. Graener, Phys. Rev. B 79, 115411 (2009).

[62] K. F. MacDonald, Z. L. Sámson, M. I. Stockman, and N. I. Zheludev, Nat. Photonics 3, 55 (2008).

[63] P. Dombi, A. Hörl, P. Rácz, I. Márton, A. Trügler, J. R. Krenn, and U. Hohenester, Nano Lett. 13, 674 (2013).

[64] M. F. Ciappina, S. S. Aćimović, T. Shaaran, J. Biegert, R. Quidant, and M. Lewenstein, Opt. Express 20, 26261 (2012).

[65] J. A. Pérez-Hernández, M. F. Ciappina, M. Lewenstein, L. Roso, and A. Zaïr, Phys. Rev. Lett. 110, 053001 (2013).

[66] S. Choi, M. F. Ciappina, J. A. Pérez-Hernández, A. S. Landsman, Y. J. Kim, S. C. Kim, and D. Kim, Phys. Rev. A 93, 021405(R) (2016).

[67] T. Shaaran, M. F. Ciappina, and M. Lewenstein, Phys. Rev. A 86, 023408 (2012).

[68] I.-Y. Park, S. Kim, J. Choi, D.-H. Lee, Y.-J. Kim, M. F. Kling, M. I. Stockman, and S.-W. Kim, Nat. Photonics 5, 677 (2011).

[69] F. C. Masim, M. Porta, W.-H. Hsu, M. Nguyen, T. Yonezawa, A. Balcytis, S. Juodkazis, and K. Hatanaka, ACS Photonics 3, 2184 (2016).

[70] K. Yoshioka, I. Katayama, Y. Minami, M. Kitajima, S. Yoshida, H. Shigekawa, and J. Takeda, Nat. Photonics 10, 762 (2016).

[71] S. E. Irvine and A. Y. Elezzabi, Appl. Phys. Lett. 86, 264102 (2005)

[72] S. Sederberg and A. Y. Elezzabi, Phys. Rev. Lett. 113, 167401 (2014).

[73] D. Bar-Lev and J. Scheuer, Phys. Rev. Spec. Top.-Accel. Beams 17, 121302 (2014).

[74] L. Fedeli, A. Sgattoni, G. Cantono, D. Garzella, F. Réau, I. Prencipe, M. Passoni, M. Raynaud, M. Květoň, J. Proska, A. Macchi, and T. Ceccotti, Phys. Rev. Lett. 116, 015001 (2016).

[75] E. A. Peralta, K. Soong, R. J. England, E. R. Colby, Z. Wu, B. Montazeri, C. McGuinness, J. McNeur, K. J. Leedle, D. Walz, E. B. Sozer, B. Cowan, B. Schwartz, G. Travish, and R. L. Byer, Nature (London) 503, 91 (2013).

[76] T. Ceccotti, V. Floquet, A. Sgattoni, A. Bigongiari, O. Klimo, M. Raynaud, C. Riconda, A. Heron, F. Baffigi, L. Labate, L. A.
Gizzi, L. Vassura, J. Fuchs, M. Passoni, M. Kveton, F. Novotny, M. Possolt, J. Prokupek, J. Proska, J. Psikal, L. Stolcova, A. Velyhan, M. Bougeard, P. D’Oliveira, O. Tcherbakoff, F. Réau, P. Martin, and A. Macchi, Phys. Rev. Lett. 111, 185001 (2013).

[77] D. Margarone, O. Klimo, I. J. Kim, J. Prokupek, J. Limpouch, T. M. Jeong, T. Mocek, J. Psikal, H. T. Kim, J. Proska, K. H. Nam, L. Stolcova, I. W. Choi, S. K. Lee, J. H. Sung, T. J. Yu, and G. Korn, Phys. Rev. Lett. 109, 234801 (2012).

[78] J. A. Sánchez-Gil and A. Maradudin, Opt. Lett. 28, 2255 (2003).

[79] J. A. Sanchez-Gil and A. Maradudin, Opt. Express 12, 883 (2004).

[80] D. G. Baranov, S. V. Makarov, V. A. Milichko, S. I. Kudryashov, A. E. Krasnok, and P. A. Belov, ACS Photonics 3, 1546 (2016).

[81] S. Makarov, S. Kudryashov, I. Mukhin, A. Mozharov, V. Milichko, A. Krasnok, and P. Belov, Nano Lett. 15, 6187 (2015).

[82] G. V. Hartland, Chem. Rev. 111, 3858 (2011).

[83] L. Novotny and B. Hecht, Principles of Nano-Optics, Vol. 1 (Cambridge University Press, Cambridge, 2006).

[84] M. I. Stockman, Opt. Express 19, 22029 (2011).

[85] H. U. Yang, J. D’Archangel, M. L. Sundheimer, E. Tucker, G. D. Boreman, and M. B. Raschke, Phys. Rev. B 91, 235137 (2015).

[86] R. Carminati, J. J. Greffet, C. Henkel, and J. M. Vigoureux, Opt. Commun. 261, 368 (2006).

[87] S. Albaladejo, R. Gómez-Medina, L. S. Froufe-Pérez, H. Marinchio, R. Carminati, J. F. Torrado, G. Armelles, A. GarcíaMartín, and J. J. Sáenz, Opt. Express 18, 3556 (2010).

[88] M. Quinten, Optical Properties of Nanoparticle Systems: Mie and Beyond (Wiley-VCH Verlag GmbH \& Co. KGaA, Weinheim, 2011).

[89] M. I. Tribelsky and A. E. Miroshnichenko, Phys. Rev. A 93, 053837 (2016).

[90] V. Giannini, Y. Francescato, H. Amrania, C. C. Phillips, and S. A. Maier, Nano Lett. 11, 2835 (2011).

[91] Y. Bao, Z. Hu, Z. Li, X. Zhu, and Z. Fang, Small 11, 2177 (2015). 